\title{
JUSTIFICATION OF THE MAPPING APPROACH FOR FINITE ELEMENT MODELLING OF DUCTILE TEARING
}

\author{
S. Hertelé ${ }^{1}$, W. De Waele ${ }^{2}$, R. Denys ${ }^{2}$ and M. Verstraete ${ }^{2}$ \\ ${ }^{1}$ FWO Aspirant, Ghent University, Laboratory Soete, Belgium \\ ${ }^{2}$ Ghent University, Laboratory Soete, Belgium
}

\begin{abstract}
Ductile tearing plays a major role in the failure behaviour of flawed pipeline girth welds under plastic deformation. Different approaches exist to describe tearing in finite element analysis, each of which has specific advantages and disadvantages. This paper focuses on the highly pragmatic mapping approach, which interpolates between results of simulations with different but fixed flaw depths. The main advantage of mapping is its straightforward connection with experimentally determined crack growth resistance curves, by application of the tangency approach. Since mapping is unique in that it does not incorporate ductile tearing within a single simulation, its physical relevance may be questioned. This paper addresses a justification of the mapping approach from a fundamental point of view. First, an analytical proof of the concept is given based upon the mathematical background of the $J$ integral. Then, a numerical validation gives confidence in the justification. Finally, attention is drawn to possible practical implementations of the mapping method.
\end{abstract}

Keywords: ductile tearing; mapping; tangency criterion; $J$ integral; plastic wake

\section{INTRODUCTION}

Due to the exhaustion of fossil fuel resources, an increasing number of pipelines is installed in harsh environments that may impose global plastic deformations to the pipeline (permafrost, landslide prone terrains, ...). The tensile strain capacity of such pipelines is to a great extent influenced by the structural response of the girth welds that connect different pipe sections. In many cases, failure is governed by the occurrence of (stable) ductile tearing, which promotes collapse of the remaining crack ligament.

Many structural integrity assessments involve the use of finite element modelling. If flawed girth welds of plastically deformed pipelines are considered, the above indicates that the incorporation of ductile tearing is essential. Different methods exist to this purpose, all of which have their strengths and weaknesses. A brief overview is provided in Section 2.

Following, this paper focuses on the justification (Section 3) and validation (Section 4) of one specific modelling approach, where ductile tearing is seen rather as a succession of simulations with different, but fixed, crack sizes. Finally, conclusions are drawn in Section 5.

\section{DUCTILE TEARING IN FINITE ELEMENT MODELLING}

Many methods have been implemented to incorporate ductile tearing in finite element analyses. This section briefly covers four commonly applied techniques, with an emphasis on their (dis)advantages: damage modelling, cohesive zone modelling, extended finite element modelling, and the mapping approach.

\subsection{Damage modelling: the Gurson-Tvergaard-Needleman (GTN) model}

Damage modelling aims to describe failure through the gradual development of damage. The most widely applied damage model for ductile tearing is the Gurson-Tvergaard-Needleman (GTN) model, which has its foundations in [1-3]. It is based on the physical process behind ductile tearing, i.e. the nucleation, growth and eventual coalescence of voids. These voids can be unmistakably observed with post-mortem macrography (Figure 1).

An unmistakably clear advantage of the GTN model is its basis of microstructural physics, which significantly increases its possibilities. For example, the path of ductile tearing can be predicted, and effects of constraint [4] and residual stresses [5] can be directly taken into account. However, the GTN model also involves some disadvantages, notably: 
- A total of eight parameters is required, which hampers its practical applicability. In particular, it may be challenging to relate the GTN model parameters to an experimental crack growth resistance curve.

- The result is highly mesh dependent. Apart from this, a very fine mesh is needed which drastically increases computational time.

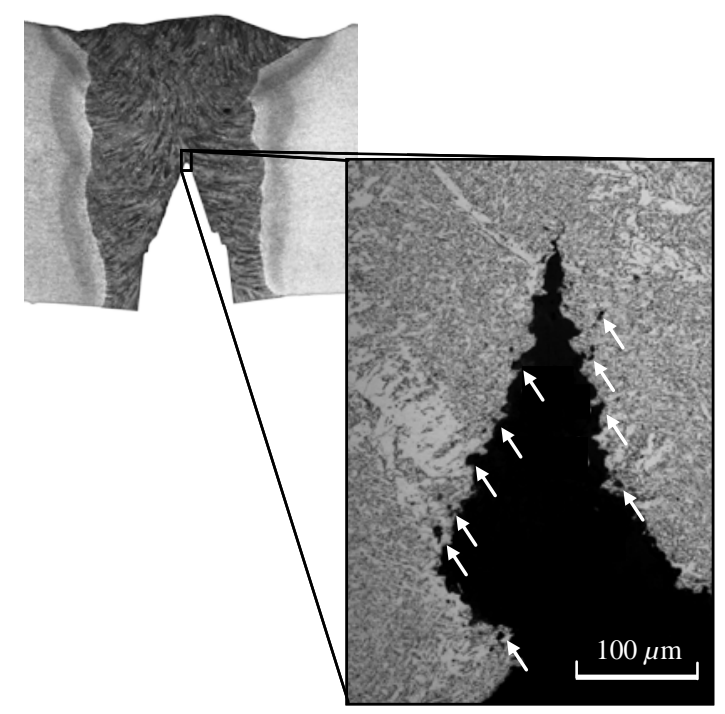

Figure 1: Ductile tearing results from the nucleation, growth and eventual coalescence of voids.

\subsection{Cohesive zone modelling}

Cohesive zone modelling assumes a pre-defined crack propagation zone ('process zone') ahead of the crack tip, in which zero-thickness cohesive elements are added as an interface between continuum elements. These cohesive elements are characterized by a 'separation law', which correlates the tensile stress with a certain separation. Throughout time, many linear and non-linear separation laws have been developed, each with a different set of characteristic parameters [6].

The application of cohesive zone modelling is attractive as its algorithm is fairly straightforward and intuitive from an engineering point of view. Nevertheless, some objections can be made:

- It is challenging to estimate the separation law parameters that comply with experimentally measured fracture behaviour. To obtain a good representation of reality, inverse modelling is required [6]. As such, much of the intuitiveness is lost.

- In the most straightforward application of cohesive modelling, a crack path is prescribed and cohesive elements are added in this prescribed path only. As a consequence, crack path predictions become impossible. To overcome this anomaly, cohesive elements can be added at the interfaces of all elements in a volume rather than a surface. However, this involves other issues such as the requirement of a very fine and computationally intensive mesh [7].

\subsection{Extended finite element modelling (X-FEM)}

The extended finite element modelling (X-FEM) method, first reported in 1999 by Belytschko and Black [8], addresses the limitation of other methods such as cohesive zone modelling, that crack propagation should follow element edges (2D) or surfaces (3D). In X-FEM, the crack is allowed to intersect edges of elements, whose nodes are enriched with Heaviside degrees of freedom to allow for discontinuous displacement jumps.

Major advantages of the X-FEM approach are that mesh dependency is strongly reduced, and that crack propagation need not follow a pre-described path. As a consequence, it can be used to predict crack propagation direction in a continuous way. 
The major limitation of X-FEM, however, is that its implementation is up to now either based upon linearelastic fracture mechanics [9] or on cohesive separation laws [10]. Whereas an application of the former is mostly restricted to fatigue crack propagation, the latter has objections similar to those of cohesive zone modelling (requirement of inverse modelling).

\subsection{The mapping approach}

The mapping approach is a simple, pragmatic alternative to the abovementioned approaches which all incorporate ductile tearing within the simulation itself. Mapping simply interpolates between results of simulations with fixed but different crack sizes. Figure 2 illustrates this approach for deformation-controlled loading, assuming $J$ as a crack driving force measure. $J$ is calculated as a function of the applied deformation $u$ (a). By plotting all results over the tearing resistance ('J-R') curve of the material under the occurring constraint conditions, and connecting points of equal deformation $u_{i}$ (b), crack growth can be estimated as a function of $u$ and failure can be identified as the point where the curves of applied $J$ and resistance tangentially touch at one point (tangency approach).

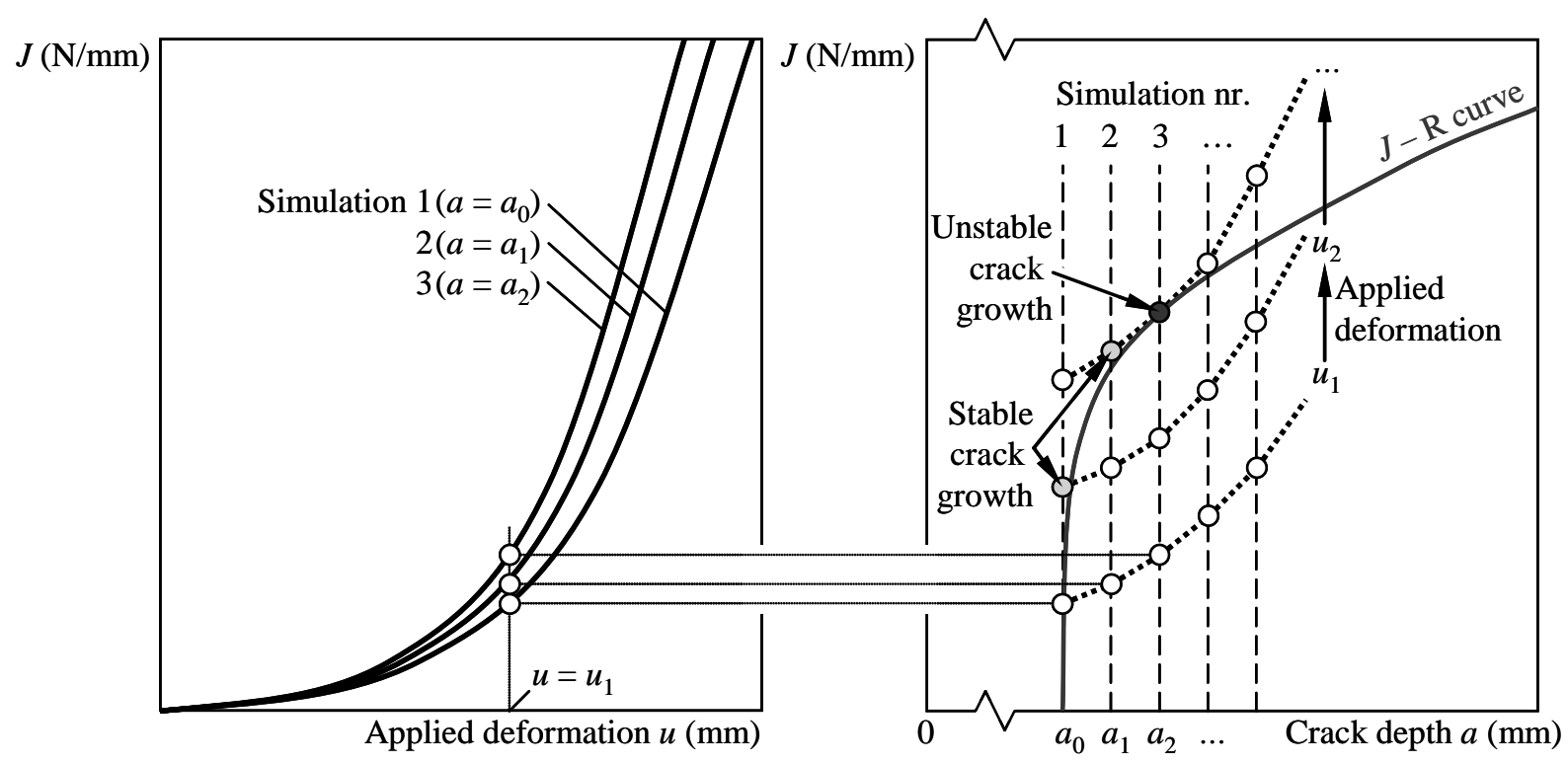

(a)

(b)

Figure 2: Application of the mapping approach (a) according to the tangency criterion (b).

The mapping approach is highly pragmatic as it directly uses an experimentally measured crack growth resistance curve. As such, no model parameters have to be tuned by inverse modelling.

On the other hand, the mapping approach involves some challenges:

- In contrast with the GTN damage model, effects of constraint are not explicitly described and should be incorporated by the input of a representative J-R curve.

- The direction of crack propagation is not predicted during the simulation itself and, therefore, requires a user-defined criterion.

The mapping approach has been widely applied in the field of strain-based design of flawed girth welds because of its high pragmatism. For instance, the recently developed strain capacity equations of Kibey et al. [11] rely on the technique of mapping.

Given its importance and common application, the remainder of this paper is fully devoted to the mapping approach. In particular, since the mapping approach does not model ductile tearing within a simulation itself, attention is given to a proper justification of its applicability. 


\section{ANALYTICAL JUSTIFICATION OF THE MAPPING APPROACH}

For the following, focus is put on analyses where $J$ is used to express driving force. As a first step towards the answer, a distinction has to be made between two different methods to calculate $J$. The following is taken from [12] and uses symbols that are commonly applied for small-scale fracture mechanics specimens (SENB, SENT): $B$ is the specimen width, $W$ the specimen thickness, a the crack depth and $b=W-a$ the crack ligament. Additionally, $B_{N}$ is the net specimen width, which is smaller than $B$ if side grooves are present.

A first method ('deformation' $J$ ) starts from the energy release rate definition of $J$ which - in absence of crack growth - can be transformed to the following relation for the plastic component $J_{p l}$ [13]:

$$
J_{p l}=\frac{\eta U_{p l}}{B_{N} b}
$$

where $\eta$ is a tabulated proportionality factor (-) which is assumed to depend only on the relative crack ligament, $b / W$. Note that, in contrast with this assumption, $\eta$-factors have been observed to additionally depend on strain hardening behaviour [14]. Further, $U_{p l}$ (N.mm) is the energy that follows from integration of the load - plastic displacement $\left(P-u_{p l}\right)$ curve:

$$
U_{p l}=\int_{0}^{u_{p l}} P \mathrm{~d} u_{p l}
$$

A second method ('far-field' J) uses Rice's [15] path-independent contour integral definition of $J$ as defined by:

$$
J=\int_{\Gamma}\left(w \mathrm{~d} y-\sigma_{i j} n_{j} \frac{\partial u_{i}}{\partial x} \mathrm{~d} s\right)
$$

where ds is a small increment along the contour $\Gamma$, taken around the crack tip as in Figure $3, x$ the orientation of the crack in a $x-y$ coordinate system, $n_{j}$ the components of the normal to this contour, and $w$ the strain energy density:

$$
w=\int_{0}^{\varepsilon_{i j}} \sigma_{i j} \mathrm{~d} \varepsilon_{i j}
$$

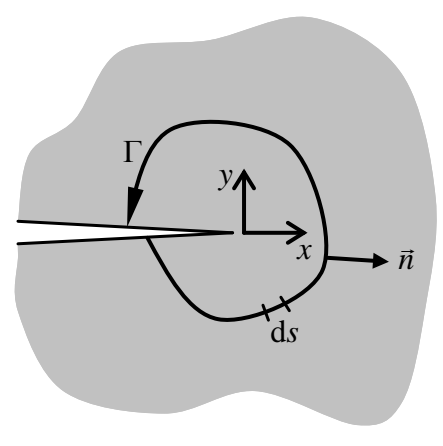

Figure 3: Contour integral for calculation of far-field $\mathrm{J}$.

In Eqs. (3) and (4), $\varepsilon_{i j}(-)$ and $\sigma_{i j}(\mathrm{MPa})$ are the tensor components of strain and stress, respectively, in the $x-y$ coordinate system.

Deformation $J$ and far-field $J$ calculations yield identical results in absence of crack growth. If ductile tearing occurs, however, a plastic wake is formed which will influence far-field $J$ but not deformation $J$. Indeed, deformation $J$ (Eq. (1)) is based upon deformation plasticity which assumes non-linear elastic behaviour rather than plastic behaviour. This assumption eliminates any strain history dependence, and a concrete consequence hereof is that deformation plasticity does not predict a plastic wake. On the other hand, the far-field J contour integral (Eq. (3)) is readily influenced by the stress-strain distribution near the crack tip. 
Additionally problematic is that a plastic wake may influence the convergence of Eq. (3), as its path independence relies on deformation plasticity.

Given the above, it is necessary - when using the tangency criterion - to compare similar formulations for $J$ in crack driving force curves and the resistance curve. To this respect, experimentally determined $\mathrm{J}-\mathrm{R}$ curves rely on the 'deformation' definition of $J$. In particular, the globally used ASTM E1820 standard [16] (SENB tests) involves the following equation to account for ductile tearing in the calculation of updated plastic $J$-values $J_{p l, i}$ from the previously measured value $J_{p l(i-1)}$ :

$$
J_{p l(i)}=\left[J_{p l(i-1)}+\frac{\eta_{i-1}}{B_{N} b_{i-1}}\left(U_{p l(i)}-U_{p l(i-1)}\right)\right]\left(1-\gamma_{i-1} \frac{a_{i}-a_{i-1}}{b_{i-1}}\right)
$$

with $\gamma$ a dimensionless factor that directly follows from $\eta(b / W)$ :

$$
\gamma=\eta-1+\frac{1}{\eta} \frac{b}{W} \frac{\mathrm{d} \eta}{\mathrm{d}(b / W)}
$$

Note that Eq. (6) involves an approximation. Hence, accuracy may be lost when crack growth occurs $\left(a_{i}-\right.$ $\left.a_{i-1} \neq 0\right)$.

Recent procedures for SENT testing $[14,17]$ adopt relations similar to Eq. (5), but with $U_{p l}$ the plastic surface below the load-CMOD curve rather than the load-displacement curve. It was found that this modification produces $\eta$-factors which are much less sensitive to $b / W$ and material behaviour.

It is shown in $[12,18]$ that Eq. (5) relies upon Eq. (1), where it is assumed that the actual crack size $a_{i}$ has been constant during the test. Therefore, Eq. (5) indeed represents a 'deformation' J. Recognition of this fact is a justification of the mapping method. Indeed, although based on the far-field equation Eq. (3) in most finite element software packages, the crack driving force curves determined with the mapping method correspond with a deformation $\mathrm{J}$ as no plastic wake is modelled due to the absence of crack growth within each simulation.

\section{NUMERICAL VALIDATION}

To validate the similarity between experimental $J-R$ curve determinations and the mapping approach, a two-dimensional (plane strain) finite element model of a clamped SENT specimen has been developed using ABAQUS ${ }^{\circledR}$ version 6.9 (Figure 4). The objective of this model is to compare $J$ integral predictions using the CANMET procedure from [17] with differently obtained numerical J-values. Assumed dimensions and material properties are given in Table 1. Note that the length-to-thickness ratio of the specimen is 10 as advised in the CANMET procedure. It was chosen to model the crack as infinitely sharp, with an uncollapsed crack tip mesh. Material was modelled using a small-strain formulation with incremental plasticity according to the Ramberg-Osgood equation:

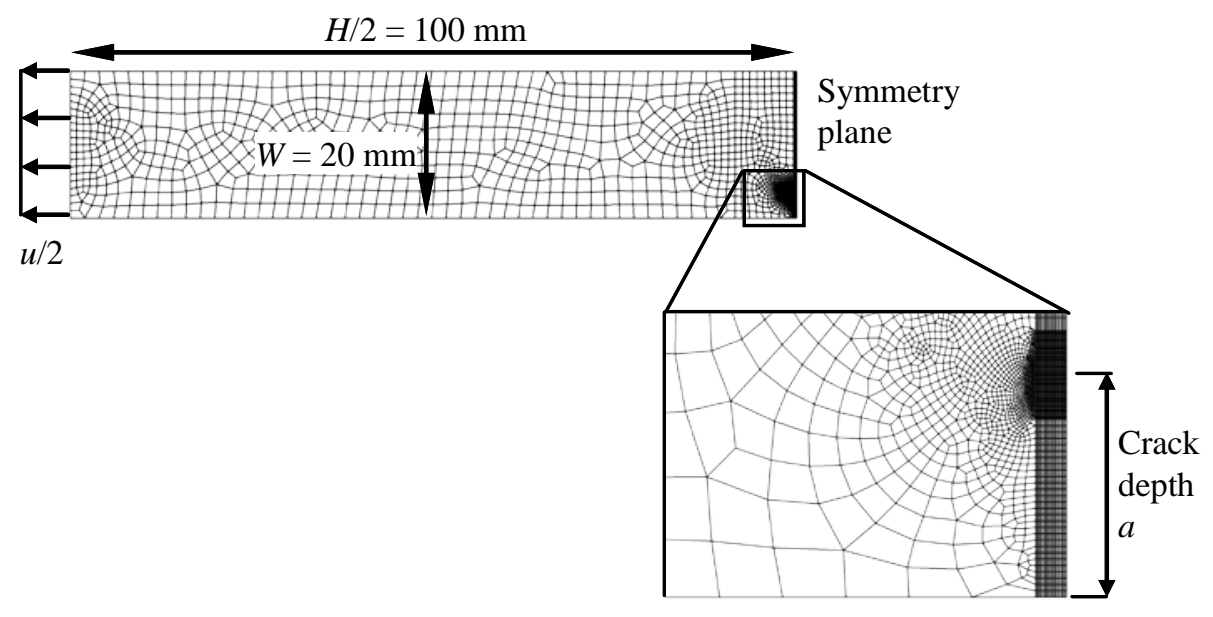

Figure 4: Geometry and mesh of the investigated finite element model. 


$$
\varepsilon=\frac{\sigma}{E}+0.002\left(\frac{\sigma}{\sigma_{0.2}}\right)^{n}
$$

Table 1: Parameter values assumed for the validation simulation

\begin{tabular}{ccc}
\hline Parameter & Symbol (dimension) & Value \\
\hline Specimen length & $H(\mathrm{~mm})$ & 200 \\
\hline Specimen thickness & $W(\mathrm{~mm})$ & 20 \\
\hline Initial crack depth & $a_{0}(\mathrm{~mm})$ & 3 \\
\hline Young's modulus & Material parameters & 200000 \\
\hline 0.2\% proof stress & $E(\mathrm{MPa})$ & 500 \\
\hline Strain hardening exponent & $\sigma_{0.2}(\mathrm{MPa})$ & 10
\end{tabular}

Only half of the specimen has been modelled due to symmetry, using a total of 4618 eight-node quadrilateral elements with reduced integration. It was arbitrarily chosen to describe an applied total displacement $u$ of $1.0 \mathrm{~mm}$ without ductile tearing, after which a crack growth of $1.0 \mathrm{~mm}$ occurs over an additional applied displacement of $0.2 \mathrm{~mm}$. Two different analysis types were performed:

- crack growth in one simulation, by releasing nodes ahead of the crack tip in ten steps. Each step, the applied displacement increases with $0.02 \mathrm{~mm}$ and the crack grows with $0.1 \mathrm{~mm}$. This method is to some extent related to the cohesive zone modelling approach.

- the mapping approach, with crack growth increments of $0.1 \mathrm{~mm}$ (ten different simulations with fixed crack depths $3.0 \mathrm{~mm}, 3.1 \mathrm{~mm}, \ldots, 4.0 \mathrm{~mm}$ ). Corresponding imposed displacements were respectively $1.0 \mathrm{~mm}, 1.2 \mathrm{~mm}$, and so on.

$J$ integral has been extracted over twenty contours around the crack tip. In the following, attention is given to values from the $15^{\text {th }}$ and the $20^{\text {th }}$ contour which is sufficient to reveal the trends of interest.

First focussing on the numerically observed J-response before ductile tearing (evidently equal for both investigated analysis types), there clearly is a strong agreement with $\mathrm{J}$-values predicted from the CANMET procedure (Figure 5(a)). This gives confidence to both the numerical accuracy of the finite element model and the analytical accuracy of the CANMET equations.

More interesting, however, is the comparison of J-values observed during ductile tearing (Figure 5(b)). Whereas the simulations from the mapping approach have a strong $J$ integral convergence (values from the $15^{\text {th }}$ and $20^{\text {th }}$ contour are nearly identical), the simulation where nodes are released suffers from significant contour divergence. This observation is related to the fact that Eq. (3) loses its path independence if principles of deformation plasticity are violated. Indeed, a plastic wake is clearly observed (Figure 6). Apart from the superior convergence properties, the simulations from the mapping approach are significantly more representative with respect to the prediction from the CANMET procedure. A small difference is observed between both, which might be appointed to the approximate character of $\gamma$ (Eq. (6)) as explained above. 


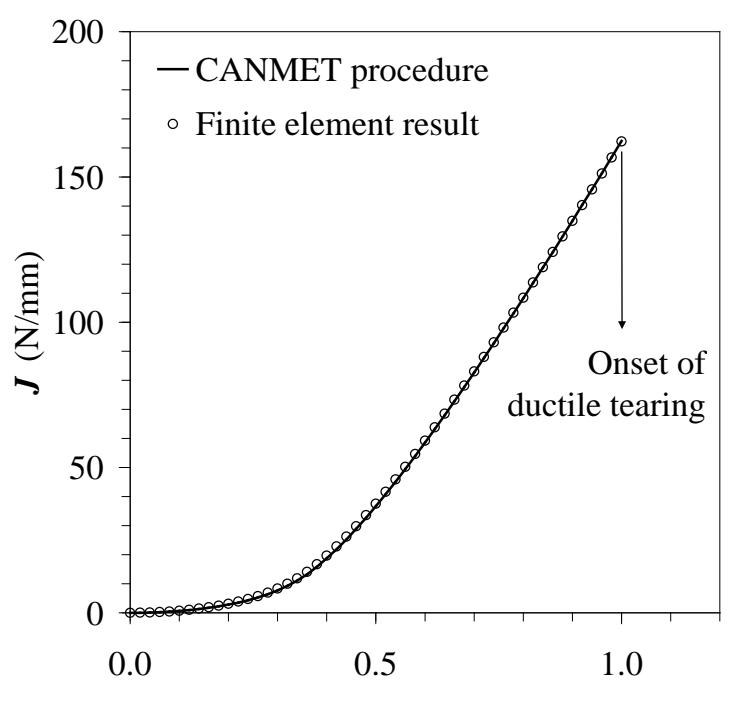

Applied displacement $\boldsymbol{u}(\mathrm{mm})$

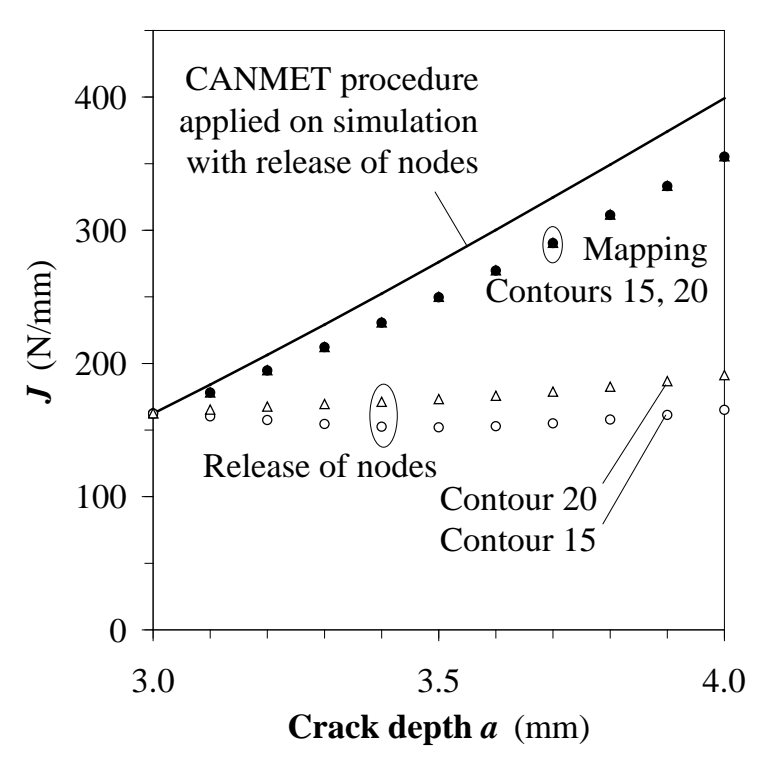

(b)

Figure 5: Comparison of $J$ integral values (a) in absence of ductile tearing, and (b) with ductile tearing.

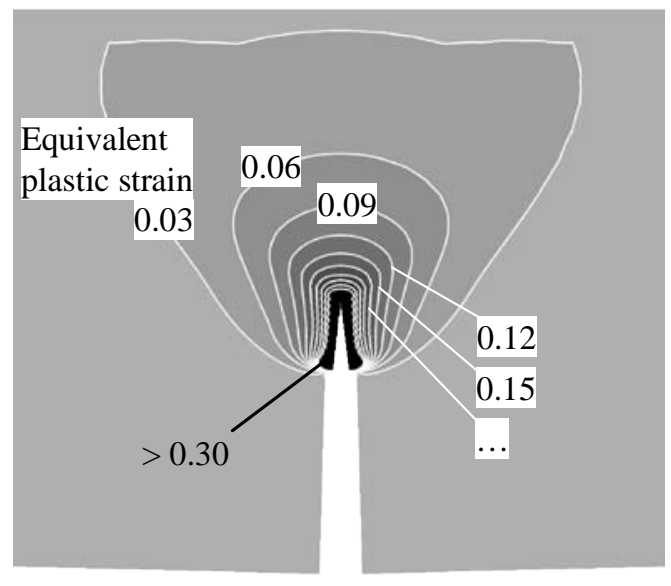

Figure 6: A plastic wake is observed around the zone where nodes were released.

\section{CONCLUSIONS}

The presented study shows that, in combination with an experimentally determined $\mathrm{J}$-R curve that represents the constraint level of the structure, the mapping approach can be validly adopted as a technique to model ductile tearing. This technique eliminates many of the issues that arise if tearing is modelled in the simulation itself, such as a strong mesh dependency, the requirement of a very fine mesh, and the necessity of inverse modelling.

The justification of the mapping approach follows from the observation that both experimental J-R curves and modelled $J$ integral responses rely upon a 'deformation' $J$ rather than a 'far-field' $J$. The former is based upon deformation plasticity, which neglects the influence of strain history present in a plastic wake. The latter is based upon a contour integral whose result and path independence is altered by this plastic wake, and is therefore unsuited for modelling applications that involve ductile tearing.

As a major challenge of the fully integrated application of the mapping approach, the necessity of a procedure to estimate the propagation path of ductile tearing can be put forward. This propagation path has been observed to be highly variable for girth weld flaws. The development and validation of such procedure is advised for future research. 
The authors would like to acknowledge the financial support of the FWO - Vlaanderen (Research Foundation - Flanders; grant nrs. 1.1.880.09.N.00 and 1.1.880.11.N.01) and the IWT Vlaanderen (Government Agency for Innovation by Science and Technology; grant nrs. SB-091512 and SB-093512).

\section{REFERENCES}

[1] Gurson, A.L., Continuum theory of ductile rupture by void nucleation and growth: Part I - Yield criteria and flow rules for porous ductile materials, Journal of Engineering Materials and Technology, 99(1), 215, 1977.

[2] Tvergaard, V., Influence of voids on shear band instabilities under plane strain condition, International Journal of Fracture Mechanics, 17(4), 389-407, 1981.

[3] Tvergaard, V., Needleman, A., An analysis of ductile rupture in notched bars, Journal of Mechanics and Physics of Solids, 32(6), 461-490, 1984.

[4] Xu, J., Zhang, Z.L., Østby, E., Nyhus, B., Sun, D.B., Effects of crack depth and specimen size on ductile crack growth of SENT and SENB specimens for fracture mechanics evaluation of pipeline steels, International Journal of Pressure Vessels and Piping, 86, 787-797, 2009.

[5] Ren, X.B., Zhang, Z.L., Nyhus, B., Effect of residual stresses on ductile crack growth resistance, Engineering Fracture Mechanics, 77, 1325-1337, 2010.

[6] Zerbst, U., Heinimann, M., Dalle Donne, C., Steglich, D., Fracture and damage mechanics modelling of thin-walled structures - An overview, Engineering Fracture Mechanics, 76, 5-43, 2009.

[7] Scheider, I., Brocks, W., Simulation of cup-cone fracture using the cohesive model, Engineering Fracture Mechanics, 70(14), 1943-1961, 2003.

[8] Belytschko, T., Black, T., Elastic crack growth in finite elements with minimal remeshing, International Journal for Numerical Methods in Engineering, 45, 601-620, 1999.

[9] Giner, E., Sukumar, N., Tarancón, J.E., Fuenmayor, F.J., An Abaqus implementation of the extended finite element method, Engineering Fracture Mechanics, 76, 347-368, 2009.

[10] Song, J.H., Areias, P.M.A., Belytschko, T., A method for dynamic crack and shear band propagation with phantom nodes, International Journal for Numerical Methods in Engineering, 67, 868-893, 2006.

[11] Kibey, S., Wang, X., Minnaar, K., Macia, M.L., Fairchild, D.P., Kan, W.C., Ford, S.J., Newbury, B., Tensile strain capacity equations for strain-based design of welded pipelines, Proceedings of the $8^{\text {th }}$ International Pipeline Conference, Calbary, Canada, 2010, IPC2010-31661.

[12] Anderson, T.L., Fracture mechanics - fundamentals and applications, CRC Press LLC, $2^{\text {nd }}$ edition, 1995.

[13] Sumpter, J., Turner, C., Method for laboratory determination of $J_{c}$, ASTM STP 601, 3-18, 1976.

[14] Cravero, S., Ruggieri, C., Estimation procedure of J-resistance curves for $S E(T)$ fracture specimens using unloading compliance, Engineering Fracture Mechanics, 74, 2735-2757, 2007.

[15] Rice, J.R., A path independent integral and the approximate analysis of strain concentration by notches and cracks, Journal of Applied Mechanics, 35, 379-386, 1968.

[16] ASTM E 1820-06, Standard test method for measurement of fracture toughness, ASTM International, West Conshohocken, USA, 2006.

[17] Shen, G., Gianetto, J.A., Tyson, W.R., Measurement of J-R curves using single-specimen technique on clamped SE $(T)$ specimens, Proceedings of the $19^{\text {th }}$ International Offshore and Polar Engineering Conference, 92-99, Osaka, Japan, 2009.

[18] Ernst, H.A., Paris, P.C., Landes, J.D., Estimations on J-integral and tearing modulus $T$ from a single specimen test record, Proceedings of ASTM STP $743,13^{\text {th }}$ Fracture Mechanics Conference, 476-502, 1981. 